\title{
Grenzen der FMEA
}

Die FMEA stellt ein wirkungsvolles Instrument des präventiven Qualität- und Risikomanagements dar. Sie hat aber auch ihre Grenzen. Die FMEA baut auf dem Erfahrungswissen der Beteiligten auf und beschreibt die Zusammenhänge zwischen Fehlerfolge-Fehler-Fehlerursache. Sie kommt damit an ihre Grenzen, wenn die Teammitglieder keine klaren Zusammenhänge beschreiben können. In diesen Fällen sollte auf die Methode Design of Experiments (DoE), oder auch Statistische Versuchsplanung genannt, umgestiegen werden. Des Weiteren wird die FMEA keine neuen Erkenntnisse bringen, wenn Produkte und Prozesse einen hohen Reifegrad erreicht haben. In diesen Fällen sollten die Erfahrungen in einer Standard-FMEA zusammengeschrieben werden, auf die dann innerhalb der Projekte verwiesen wird. 